\title{
Resolución precursora y temporalidad: el horizonte originario de la mismidad en Ser y Tiempo $(\$ \S 61-66)^{*}$
}

\author{
Anticipatory Resolution and Temporality: The \\ Primary Horizon of the Selfhood in Being and \\ Time $(\$ \oint 61-66)$
}

\author{
JUAN JOSÉ GARRIDO PERIÑÁN \\ UNIVERSIDAD DE SEVILLA
}

Recibido: 23/12/2019 Aceptado:12/07/2020

\section{RESUMEN}

El presente artículo intenta explorar una relación metódica y temática entre dos existenciarios: mismidad y temporalidad, en sus respectivos límites de aparición, en una obra de gran complejidad como Ser y Tiempo, a fin de poder captar, con la radicalidad que solo la fenomenología permite hacer valer, la unidad integral del ser del Dasein, un ente caracterizado y señalado, a diferencia de los demás entes intramundanos, por la existencia de un modo raigal, ontológico. La conclusión apuntará a vertebrar el análisis existencial en una experiencia primaria como la del sí-mismo.

$$
\text { PALABRAS CLAVE }
$$

FENOMENOLOGÍA, TEMPORALIDAD, RESOLUCIÓN, MISMIDAD

*Artículo elaborado al socaire del Proyecto I+D De Excelencia (FFI2017/83770-P): "Dinámicas del cuidado y lo inquietante. Figuras de la inquietante en el debate fenomenológico contemporáneo y las posibilidades de una orientación filosófica. Configuración teórica y metodológica", financiado por el Ministerio de Ciencias, Innovación y Universidades del Reino de España. 


\section{ABSTRACT}

This paper tries to explore a methodical and thematic relationship between two existentials: Selfhood and Temporality, as they are brought to light through the existential anlysis of Dasein in Martin Heidegger's complex and radical work Being and Time. The goal of this paper is to grasp in a radical way that only the phenomenological approach makes possible the comprehensive unity of the being of Dasein. Dasein is the only being that is characterized, unlike any other being, by the existence of an extreme and ontological mode. The conclusion will aim to support the existential analysis in a prime experience of the self.

\section{KEYWORDS}

PHENOMENOLOGY, TEMPORALITY, RESOLUTION, SELFHOOD

\section{INTRODUCCIÓN}

COMO SE DEJÓ VER EN PUBLICACIONES anteriores, ${ }^{1}$ la mismidad del Dasein, en tanto modalidad en correlación con la «verdad de la existencia» ${ }^{2}$ [ Wahrheit des Existenz] -entendida ésta como un modo de apertura autorreferencial y en homologación con la «propiedad» [Eigentlichkiet] de la existencia-, constituía una condición de posibilidad necesaria y suficiente, sobre todo con la vista puesta en dos momentos del análisis fenomenológico desarrollado, a saber: en primera instancia, en relación con la tarea analítica ejercida mediante una descripción de carácter hermenéutico, desarrollada en la I Sección; y, en segunda instancia, con respecto al análisis de la propiedad, aventurada en los capítulos uno y dos de la II Sección sobre el «ser-para-la-muerte» [sein-zumTode], el «ser-total» [Ganzheit], la «conciencia» [Gewissen], el «ser-culpable»

1 Hemos investigado y desarrollado el rol metódico y temático que cumple el existenciario «mismidad» [Selbstheit], en un libro como Ser y Tiempo -en adelante SyT-, en las siguientes publicaciones: Garrido-Periñán 2019, pp. 887-911; 2019b, pp. 159-175; 2019c, pp. 411-428; 2019d, pp.175-206. En el caso de que el lector quisiera extender la temática tratada en esta publicación, la consulta de las publicaciones citadas se antoja indispensable.

2 Habría que hacer notar que la verdad de la existencia se constituye al socaire del tratamiento de la verdad llevado a cabo en el $§ 44$ (Heidegger 2002, pp. 212-130), donde el Dasein constituye el fundamento de la verdad, en tanto que el «ser-descubridor» [Entdeckend-sein] -relativo a la adecuación enunciativa de origen apofántica- y el «estado de descubierto» [Entdecktheit] -relativo a los entes intramundanos, en sus modos de acceso-penden y son deudores del «estado de abierto» [Erschlossenheit], singular y específico de este ente-Dasein. Ahora bien, en la «verdad de la existencia» ya se insinuaba la posibilidad «de la modalidad de la propiedad» [im Modus der Eigentlichkeit], pues se entendía como una acción proyectivo-ejecutiva en la que el Dasein, en su «poder-ser» [Sein-können], se constituía como su más propio sí-mismo (Heidegger 2002, p. 221). 
[Schuldigsein] y la «resolución» [Entschlossenheit]. Esto quería decir, grosso modo, que la mismidad del Dasein no era un fenómeno adyacente de cara a abordar un análisis fenomenológico sobre el opus magnum de Heidegger, sino, antes bien, un existenciario fundante ${ }^{3}$ y originario ${ }^{4}$, el cual cumplía un rol constitutivo a su trascendencia: posibilitaba la condición de posibilidad, la venida a la presencia de la posibilidad de comprensión de la propia «temporalidad» [Zeitlichkeit], específica y singular, del ente que somos nosotros mismos, de nuevo.

Y esto es, entre tanto, lo que se pretende pensar en este artículo filosófico: hasta qué punto la relación mismidad-temporalidad ha de ser comprendida en sus respectivos límites de aparición en $S y T$, a fin de poder captar, con la radicalidad que solo la fenomenología permite hacer valer, la unidad integral del ser del Dasein, un ente caracterizado y señalado, a diferencia de los demás entes intramundanos, por la existencia de un modo raigal, ontológico. Por tanto, se va a tratar de realizar un análisis de corte fenomenológico sobre el Capítulo Tercero de la II Sección de Ser y Tiempo (§§ 61-66), en virtud del tratamiento que se hace de la temporalidad, la cual queda adscrita al modo en el que ya siempre se ejecuta el sentido implícito, por temporal, del «cuidado» [Sorge], el existenciario que recogía, en palabras de Heidegger, la «totalidad» [Ganzheit] del ser del Dasein (2002, p. 252). Esta tentativa es, empero, ejercida al socaire de lo dejado atrás en el análisis de la conciencia y la resolución, pues, entre tanto, lo que estaba en juego era la posibilidad de una comprensión ontológica y existencial de la manera en la que el Dasein, en cada caso, aparece como Dasein, es decir, en calidad de ente genuinamente ontológico, y no, más bien, como reflejo del mundo en el que vive, ${ }^{5}$ o como correlato intencional del «uno-mismo» [man-selbst]. Por esta razón, el Capítulo Tercero de la II Sección de SyT tiene como finalidad dar cuenta de hasta qué punto el cuidado rezuma tiempo. A decir verdad, esto es algo que Heidegger ya se aventuró a manifestar -sobre todo en el $\S 41-$, pero que no fue del todo concretizado en su posibilidad existencial, por cuanto el cuidado quedó indeterminado y

3 En el sentido de «fundar» [gründen], es decir, en tanto «instaurar» [stiften], «tomarsuelo» [Bodennehmen] y acto de «fundamentar» [Begründen].

4 Palabra de largo alcance en el pensar de Heidegger, también, en su determinación sustantivada «originariedad» [Ursprünglichkeit]. Que un fenómeno sea originario, en clave fenomenológica, significa la capacidad de retrotraer y/o proyectar el fenómeno eminente de tal Dasein a una mostración de carácter totalizante, atendiendo a cada una de sus estructuras intencionales en cuanto ser-en-el-mundo.

5 Sobre la «refracción ontológica» [ontologische Rückstrahlung]: «En el Dasein mismo y, con ello, en su comprensión de ser reside lo que vamos a poner de manifiesto como la refracción ontológica de la comprensión del mundo sobre la interpretación del Dasein» (Heidegger 2002, pp. 15-16). 
deshilachado en el compromiso estructural que tiene toda analítica existencial: dar cuenta de la manera en la que se ejecutan y se llevan a cabo las referencias ligadas al horizonte de comprensión que traspasa a ese ente señalado. Este fue, entre otros, unos de los motivos más importantes, los cuales sirvieron de base para el desarrollo de los análisis relativos al ser-para-la-muerte como vía afectiva, a través de la cual nosotros mismos, en tanto existentes, tenemos una primera acreditación con nuestra propia «totalidad» entendida como «fin(al)» ${ }^{6}$ [Ende]. Pero, asimismo, el ser-para-la-muerte apareció como impotente a la hora de explicar el modo de ejecución, de «ser-responsable» [verantwortlich], sobre tal relación con nuestro fin(al). Con otras palabras: el análisis del serpara(vuelto-a)-la-muerte dejó abierta la cuestión, nada baladí, de la manera en la que el Dasein tiene que llevar a cabo el poder-ser que ya siempre es.

Lo último manifestado, va a proveer el hilo conductor de los $\S \S 61-66$, o sea, el intento de Heidegger por atisbar la temporalidad sobre el cuidado vendría a responder a esta necesidad ya implícita en la caracterización de este ente como tener-que-ser y ser-en-cada-caso mío (2002, § 9), y no es, más bien, una exigencia que Heidegger sacara a relucir en desconexión con lo ya pensado y abierto en el desarrollo de su obra. Por eso, ante esta situación metódica, de cara a abordar el problema de la conexión interna, ora entre la referencia hermenéutica o «sentido de relación» [Bezugssinn] -cómo del comprender- y el «sentido de ejecución» [Vollzigssin] -cómo se «lleva a cabo» [vollzieht] lo que somos-, ora entre el ser-total abierto vía angustia a través del ser-para-la-muerte y la resolución, o sea la manera en la que asumimos nuestro fin(al), vamos a partir de la siguientes hipótesis de lectura, las cuales serán objeto de nuestra investigación, a saber: a) la mismidad, como fenómeno eminente por el que el Dasein es su «propio sí mismo» [eigene Selbst] (Heidegger 2002: 262 ss.), en términos metodológicos, permite la no «hipostatización», al modo de lo «flotante en el vacío» [freischwebende], de la vinculación de los dos momentos arriba anunciados, esto es, entre el modo hermenéutico-referencial y el relativo a la ejecución. Por este motivo, se tendrá que ver de qué manera la mismidad constituye una concretización existencial de la resolución; b) como consecuencia de lo dicho, habrá que pensar si la mismidad, en tanto «atestiguación/acreditación» [Bezeugung/Ausweisung] de nuestro genuino poder-ser, se encuentra posibilitando la manera ejecutiva de ser «en propiedad» [Eigentlichkeit] por parte del Dasein, ${ }^{7}$ pudiendo dar cuenta

6 El existenciario «ser-para-la-muerte», no ha de significar que el «fin(al)», cual destino, del existente sea morir, sino, antes bien, poner en claro que la estructura del Dasein radica, precisamente, en «tener-que-ser», en todo caso, y en hacerse cargo de aquello que es: un «poder-ser».

7 Al final del § 53, Heidegger deja abierta una cuestión (2002, p. 266): «¿Exige el Dasein, incluso sobre la base de su ser más propio, un modo propio de poder-ser que esté determinado por el precursar?». 
de la llamada «resolución precursora» [vorlaufende Entschlossenheit], como modalidad óptima de venir a presencia de la misma temporalidad ${ }^{8}$ siendo que ésta es el modo en el que el Dasein asume su propiedad desde y con la determinación existencial del «adelantarse a la posibilidad» [Vorlaufen in die Möglichkeit]; c) por último, se aventurará una tematización de la mismidad en calidad de condición de posibilidad en relación con el ser-total del Dasein, esto es, de la propia e «irrespectiva» temporalidad que somos. Esto nos llevará a realizar una mirada crítica sobre el rol que juega la experiencia de la mismidad en el $\S 63$.

\section{MisMidAD Y LA VALIDEZ DE LO ONTOLÓGICO.}

¿Se ha llegado, a través de las páginas precedentes en el libro $S y T$, a una captación originaria, fundante, del Dasein en tanto ser-total, o sea, en tanto sí-mismo, o, en cambio, los análisis anteriores solo mostraron una parte fragmentaria y azarosa del este ente peculiar, caracterizado por la compresión del ser? La respuesta debe ser ambigua, pues se ha llegado en parte sí, y en parte no. El desarrollo sobre el morir puso el dedo en la llaga, y el Dasein pudo comprobar su finitud a través de su relación necesaria con el fin(al) de su existir; por otro lado, los desarrollos en torno a la resolución ahondaron sobre la determinación de la propiedad como una modalidad relativa a la «elección» [Wahl], pero, por así decir, su conexión con el horizonte temporal, finito, abierto por el ser-para-la-muerte, no fue mostrada. En efecto, Heidegger es muy consciente de las limitaciones del análisis realizado (2002, p. 301) y del compromiso adquirido. Por esta razón, la conexión entre ambos momentos debe ser mostrada mediante la exigencia de búsqueda de la cosa misma a pensar, y no, en cambio, ser presupuesta o inoculada forzosamente. La dignidad de la fenomenología, como método óptimo de cara a abordar la relevancia de las cosas mismas, está en juego. Así, entre estos dos modos, el ser-para-lamuerte no podía cumplir las exigencias de acreditación experiencial que toda fenomenología comporta, pues la relación con nuestro fin(al) es, al menos, ambigua, soterrada y no clara. Queda entonces patente porqué Heidegger dice que es el fenómeno de la resolución la base de atestiguación fenoménica que nos permitirá profundizar sobre la conexión de los dos momentos arriba mentados (2002, p. 302). La resolución quedaba, entonces, determinada como una forma concreta de atestiguación que potenciaba el modo de ser del Dasein como un poder-ser, el cual debe ser asumido siempre ya como cuidado. Pero, en cambio, el hecho metódico que coloca a la resolución como base fenoménica, a fin de poder llevar a término el modo de presentación fenomenológica de

8 (2002, p. 304): «Como fenoménicamente originaria, la temporalidad es experimentada, en el modo de ser-total propio, en el fenómeno de la resolución precursora». 
la conexión entre los dos momentos, ser-para-la-muerte, la elección y/o el «querer-tener-conciencia» [Gewissenhabenwollen], no ha de querer decir mucho si el modo afectivo, a través el cual el Dasein «se encuentra» [befindensich] con esta resolución, no le concerniera, en sus carnes, a este mismo ente. Que le concierna o afecte significa que el poder-ser, que se resuelve a existir, siempre demanda una unidad constitutiva, no a la manera paradigmática de la yoidad, sino sobre la estructura cinética, constitutiva del «ser-en-el-mundo» [in-der-Welt-sein], la cual anhela un momento de unidad entre los horizontes de correlación de sentido, co-originarios a lo relacionado mismo: «mundoentorno» [Um-Welt $]$, «mundo-compartido» [Mit-Welt $]$ y «mundo de sí mismo» [Selbst-Welt]. Esto implicaba, en parte, afirmar la insuficiencia de los análisis previos sobre las esferas circunmundana y compartida, pues el Dasein va siempre más allá ${ }^{9}$ de una constitución cósica, en «estado de apertura» [Erschlossenheit] y en horizontalidad co-originaria ${ }^{10}$ sobre tales momentos constitutivos del ser-en-el-mundo.

\section{LA RESOLUCIÓN PRECURSORA.}

Que la resolución sea precursora debe hacernos pensar. Dicho así, aunque se revista de diferentes formas y significaciones, quizás de carácter no tan trágico, ${ }^{11}$ el Dasein se resuelve en cada caso como poder-ser, asumiéndose como posibilidad, una posibilidad determinada por la muerte. Si esto es cierto, la resolución precursora sería el acto de existir, por parte del Dasein, que recoge, en ese mismo acto, tanto lo abierto por el morir, como lo puesto en relación con la elección y/o decisión: el carácter de toda resolución es, per se, templado por la anticipación de la muerte:

La resolución llega a ser propiamente lo que ella puede ser en tanto es un comprensor ser-para-el-fin(al), esto es, como un 'pre-cursarse en la muerte' [als

9 Va más allá, pero no en la manera de quedar fuera del «comportarse» [Verhalten-sich], inherente a toda actividad entendida como ser-en-el-mundo; antes al contrario, el Dasein va más allá en virtud de su trascendencia como ente marcado por la compresión del ser del ente.

10 En definitiva, si el Dasein, en su mostración como ente señalado por la comprensión del ser, no ha de ser ni un yo, ni una substancia, ni cualquier otra modalidad de «carácter cósico» [Dinghaftigkeit], esto implica que el Dasein, de partida, se constituye como un horizonte cooriginario con lo que siempre ya se relaciona, dentro del mundo-entorno y mundo-compartido. ¿Por qué, entonces, demandar una unidad, si este ente es co-originario a sus relatas? Aquí sale a relucir la cuestión de la mismidad y su encaje en la sistematicidad inherente a SyT.

11 Mutatis mutandis, el «aburrimiento profundo» [tiefe Langeweile] jugaba, en 1930, el mismo rol temático y metódico que la angustia de 1927. La diferencia afectiva entre ambos modos de encontrarse en el mundo es evidente (Heidegger 1983, §§ 29-38). Entre las exégesis más valiosas en torno al rol que el aburrimiento juega sobre las derivas del pensamiento de Heidegger (Opilik 1993, pp. 141-196). 
Vorlaufen in den Tod]. La resolución no tiene solamente un nexo con el precursar como un otro diferente de ella misma. Ella entraña 'el ser-para-la-muerte de un modo propio' [das eigentliche Sein zum Tode], como la posible modalidad existentiva de su misma propiedad (Heidegger 2002, p. 305). ${ }^{12}$

Como se deja ver, Heidegger hace depender la resolución de un modo de asumir, comprensivo y de carácter ejecutivo, el cual tiene que erigirse como una manera de hacerse-cargo sobre el carácter de «nihilidad» [Nichtigkeit] que embarga a todo Dasein, ya que, en tanto ontológico-existencial, el modo de ser de este ente nunca es homologable a un ente intramundano. Si se mira bien, aunque las expresiones «ser-culpable» y «nada» puedan desorientarnos sobre el fondo temático que aquí está en juego, tal culpabilidad y nihilidad solo son un modo de determinación de la finitud temporal realzada por la estructura total del Dasein, el cuidado. De este modo, aquello que es asumido nunca es una nada o una culpa moral, sino la posibilidad determinada bajo el carácter de la existencialidad. Por tanto, la resolución, entendida como un acto existencial, radicado en la asunción y comprensión de lo que somos, siempre se resuelve en una determinada posibilidad, la cual queda abierta como proyecto y horizonte, y vivida como finita, a través de la cual cada Dasein tiene que asumirla a la manera de una pre-cursión (de sí). En este caso, aquello anticipado en la resolución es justamente aquella posibilidad siempre ya latente en cada ejecución que constituye el poder-ser de este Dasein: la muerte entendida como la posibilidad de toda imposibilidad. Pero esto no es todo, el poder-ser, que tiene que asumirse mediante una resolución de carácter anticipador, no es pensado bajo el orden de una posibilidad abstracta o teorética, sino que tiene que asumirse y realizarse en la guisa de una posibilidad fáctica, concreta. El ser que se presta a la resolución es el ser del Dasein mismo, no otro. Podrá entenderse, ahora con más vigor, la participación constitutiva del pre-cursar de la muerte sobre la resolución: el ser-para-la-muerte hace posible una Kehre en la resolución, modalizándola en su respectiva propiedad. Y esto en virtud del tratamiento que se ha ejercido con anterioridad sobre el «uno-mismo», el cual tiene el cariz de una estructura de emplazamiento, que no solo dispone al Dasein dentro de concepciones hermenéuticas aptas para desarrollar su existencia en el mundo, sino que le priva de un acceso afectivo-comprensivo a su modo de ser, entendido como un poder-ser que ha de relacionarse con su fin(al). Solo así se puede entender, no solo la crítica al das Man, como forma correlativa de la impropiedad del existir, sino el rol tan importante que juega el ser-para-a-la-muerte a la hora de poder conectar resolución precursora y propiedad, pues la propiedad del Dasein siempre implica una aceptación, en

12 Comillas nuestras. 
el orden de la posibilidad fáctica de su ser, de su propio fin(al): «Tan solo la resolución precursora comprende el poder-ser-culpable de forma propia y completa, es decir, originaria» (Heidegger 2002, p. 306).

Ahora bien, algo antes, Heidegger dijo que el ser-para-la-muerte era la posibilidad «más propia» [eigenst], «irreferible» [unbezüglich], «insuperable» [äußerst], «cierta» [gewiß] e «incierta» [ungewiß] (2002, p. 250), y no estaba alardeando de epítetos para mostrar el drama de la vida, sino, justamente, la intención heideggeriana radiacaba en mostrar el rol que este existenciario juega en la analítica existencial, a fin de poder dar respuesta a la pregunta por el «quién» [Wer] del Dasein, entendida ésta en los términos de su mostración fenomenológica. Las adjetivaciones sobre el ser-para-la-muerte que más han llamado la atención a los intérpretes, por lo general, fueron aquellas que enlazan certeza e indeterminación ¿Cómo es posible, pues, que la muerte sea cierta e indeterminada, más allá del ejemplo manido de que sabemos que nos moriremos, pero es imposible, al contrario, determinar el cuándo? Creemos no equivocarnos al afirmar que es la resolución precursora la que da respuesta a la aparente paradoja sobre estos dos momentos: certeza e indeterminación. Y no es solo la constatación, de carácter cotidiano, de que el Dasein muere indeterminado, como dice Heidegger poéticamente: «muere como nacido» [gebürtig stirbt] (2002, p. 374) y, sin embargo, cierto de su propio fin(al), sino que la muerte, en su modalidad de ser-para-la-muerte es un momento co-originario y estructural de la propia resolución, y no un momento externo, impuesto desde fuera. Si la resolución es, siempre ya, precursora, la muerte, como modalidad con nuestro fin(al), no puede emanar allende, sino que debe ser parte integral de nuestro modo de ser, siendo, entonces, que la «resolución» [Entschlossenheit] es la pura y nuda «verdad de la existencia», o sea, el modo excepcional por el que la existencia es dada a sí misma, mostrada en tanto tal, como cosa misma, apareciendo en tanto existencia, en su «estado de apertura» [Erschlossenheit $]$.

Podrá entenderse mejor, entonces, la relación entre certeza y muerte: el Dasein estará cierto de su modo de ser, basado en el estado de apertura, si es capaz de mantener y conservar su modo de existir a través de una resolución que se decida a asumir su fin(al). Es, en concreto, a lo que se refiere Heidegger cuando habla de «revocación» ${ }^{13}$ [Zurücknahme], o sea, al correlato de una resolución que mantiene y conserva abierta, en una situación concreta, aquí y ahora, el estado de apertura del Dasein. Tal estado de apertura no es más que lo situado-circunstanciado de un poder-ser, el cual es llenado

13 (2002, p. 308): «La certeza del acto resolutorio significa: 'mantenerse libre para su posible y acaso fácticamente necesaria revocación'» [Sichfreihalten für seine mögliche und je faktisch notwendige Zurücknahme]. Cursivas nuestras. 
de contenido mediante una posibilidad de carácter fáctico. Que la revocación abra la situación para el estado de apertura, en término negativos, pudiera inferirse que esta revocación cancela las posibilidad englobadas en el modo de ser del Dasein, ya sean éstas entendidas desde hechos pasados afincados en la conciencia, o desde hechos históricos datados en una historia; pero, antes al contrario, la revocación es del acto de ejecutar lo que somos en el modo de abrir una situación (finita), la cual queda tematiza a través de un intento por preservar la apertura, el «dejar-libre» [freigeben] a la posibilidad, que es el Dasein mismo, para que este ente pueda «re-tomarse a sí mismo» [Wiederholung ihrer selbst]. La revocación es la vía por la que la mismidad del Dasein puede, en cada caso, ser asumida y, por ende, tematizada, por la sencilla razón de que, a través de una situación, producto de una resolución de tonalidad anticipativa, la posibilidad ha quedado abierta y preservada como algo que es propio y singular de este ente-Dasein. Esta conexión, por lo demás, entre revocación y mismidad, no es nueva de este Capítulo, sino que ya fue desarrollada antes, cuando Heidegger, en la I Sección, trató la temática del sí-mismo bajo el criterio de la pérdida de sí (2002, pp. 126-130):

Precursoramente resuelto, el Dasein se mantiene abierto para la incesante pérdida en la irresolución del uno, la cual amenaza desde el fondo de su propio ser.' 'La irresolución se conoce como la incesante posibilidad, concomitantemente cierta, del Dasein' [Die Unentschlossenheit ist als ständige Möglichkeit des Daseins mitgewiß] (2002: 308). ${ }^{14}$

Es decir, la revocación permite tematizar el sí-mismo en tanto modalidad impropia: el uno-mismo, haciendo ver sus limitaciones e insuficiencias, por cuanto se ha señalado una conexión inextricable entre resolución precursorarevocación-mismidad:

El precursar no es un comportamiento flotante en el vacío, sino que debe entenderse como la posibilidad de su estado de propiedad escondido en la resolución existentivamente atestiguada, la cual debe quedar co-atestiguada también ella misma (2002, p. 309).

Ahora bien, esta triple relación -entre resolución precursora, revocación y mismidad- es, in nuce, problemática, empezando por la demanda de acreditación experiencial sobre la resolución de carácter precursor, pues, como se ha dejado ver, la modulación llamada precursora sobre la resolución, solo se ejercía al socaire de la relevancia relacional del Dasein con su fin(al). Según parece, desde el tratamiento de la resolución precursora se produce una

14 Comillas nuestras. 
dislocación entre los momentos metódicos nucleares, los cuales han guiado, hasta el momento, la posibilidad de reconversión fenomenológica de lo óntico sobre lo ontológico, a saber, desde la diferencia de nivel de análisis entre lo «existentivo» [existenzielle], en relación con lo óntico, y lo «existencial» [existenzial], con respecto a lo ontológico. En efecto, si lo ontológico era pensado como una reconducción interpretativa de la mirada, desde el modo de darse el fenómeno en la cotidianidad -no al revés-, con la resolución de carácter precursor parece que esto no sucede, al menos, en los términos planteados al inicio de SyT. Según pensamos, esta dislocación entre los momentos ónticos y ontológicos del análisis hace énfasis en la regulación de lo ontológico, el cual para ser y constituir un conocimiento «tematizable» para la fenomenología, necesita constituirse como experiencia y/o vivencia, siendo que la resolución precursora no es tan solo una posibilidad eminente, sino solo posible en tanto hecho existencial, entendido éste como «acto resolutorio» [Entschlußt] (Heidegger 2002, p. 298). De este modo, no es tan solo que el contenido existentivo provea las condiciones de posibilidad fenomenológica de la experiencia de orden ontológico, sino que la experiencia ontológica necesita invertirse sobre la experiencia de orden existentivo-óntico, de tal manera que busque no solo su concretización, sino, más bien, su validez y/o legitimidad. Por último, es aquí donde puede situarse unas de las principales funciones de la mismidad, de cara a entender este problema, no lo olvidemos, que nace como intento de dar cuenta, de una manera cabal, total e íntegra, del aparecer del Dasein qua Dasein. La mismidad, entonces, permite que la legitimidad y validez buscada no sea edulcorada con criterios ideales y abstractos, sino que permite que validez de lo ontológico sobre lo óntico sea entendida en términos de regulación con la posibilidad más propia: que el Dasein pueda ser, en cada caso, su sí-mismo.

\section{LA EXPERIENCIA DE LA MISMIDAD: LA ESTABILIDAD DEL SÍ-MISMO}

La demanda por explicitar las estructuras existenciales del Dasein, de una manera cabal e íntegra, es una tarea muy esquiva. Y ello por diversas razones, a saber: si la resolución precursora conlleva inevitablemente una indicación ante un sí-mismo en propiedad, y esta propiedad, para ser cabalmente entendida, tiene que ser abierta en una situación, como un momento propicio en el que el modo de ser del Dasein sale a relucir qua Dasein, resulta un problema la incardinación del tratamiento realizado por Heidegger en torno al uno-mismo, modalidad auto-delegatoria por la que el Dasein es nadie, el cual sirvió de base e ingrediente para preparar el tránsito de la impropiedad a la propiedad. Pero la dificultad va, todavía a más, sobre todo cuando, por atenencias metódicas se parte de la experiencia concreta y fáctica en el análisis, y se dice que el sí-mismo más propio es, precisamente, lo más lejano para con el Dasein. Es 
decir: que lo ontológicamente más propio e idiosincrático del Dasein-ser una modalidad existencial que se funda en el cuidado-es lo más lejano; o sea, nuestra mismidad es el fenómeno que más alejado está de cara a su ejecución, por un lado, y su tematización, por otro. La dificultad de la empresa aumenta cuando es pensado el sí-mismo más propio como un producto de una «modificación» [Verwandlung] de la estructura ruinosa y cadente denominada «el uno» [das Man], pues no toda forma de ir contra lo dado de antemano sería una forma de propiedad. En este sentido, la resolución precursora puede inocularse en el existir para propiciar una reinstauración de un sentido genuino, el cual se dirija en tanto contra-movimiento hacia la propiedad, pero, bien mirado, esta explicación, sigue siendo insuficiente. $\mathrm{Y}$ es insuficiente porque, como fondo posibilitador de la resolución precursora, tiene que pervivir una experiencia de orden existencial, irresolublemente asociada con el Dasein, la cual es signada por nosotros con el rótulo de mismidad. No es de extrañar que Heidegger dude, titubee y pregunte: «¿De dónde es proporcionado lo que constituye la existencia propia del Dasein?» (2002: 312). Obviamente Heidegger parece encontrarlo en la experiencia capital denominada ser-para-la-muerte, pero esta justificación es vacía, huera, carente de acreditación por la vía de la vivencia humana. Es necesario ver, entonces, el papel de la mismidad como condición de posibilidad de una tematización integral del ser del Dasein.

\section{LA MISMIDAD Y LA ESTABILIDAD.}

Heidegger afirma: «El estado de propiedad del poder-ser-sí-mismo garantiza el ver-previo en dirección a la existencialidad originaria, y ésta asegura la acuñación de los conceptos existenciales adecuados» (2002, p. 316). Esto ha de ser pensado en toda su limitación, en tanto a través del cual el Dasein aparece siendo él mismo. Ahora bien, según parece, a causa del tratamiento ejercido en el $\S 64$, la mismidad es pensada como un momento subsidiario del cuidado, o sea, es el cuidado, en tanto horizonte de temporalidad extática relativa al Dasein, el que da fundamento a la mismidad, no al revés. Aquí el análisis parece moverse en una ambigüedad, pues, prima facie, la experiencia fenoménica del yo-soy parece distar de la experiencia de la mismidad, aunque la mismidad sea vivida, siempre ya, como la vivencia de un «yo». Siguiendo el hilo conductor de la crítica heideggeriana, la cual pudiera haberse realizado a partir de un análisis de la experiencia cartesiana del cogito, incluso desde la idea de persona, relativa a la fenomenología de la empatía, lo cierto es que Heidegger solo se fija en Kant, en la teoría de la apercepción trascendental (Garrido-Periñán 2019e, pp. 9-27): el yo, cual enlace, que acompaña a todas mis representaciones, no siendo él mismo una representación. No es lugar aquí para demorarnos en esta crítica, y en las razones a través de la cuales Heidegger decide seleccionar a Kant. Según parece, para Heidegger, la determinación 
kantiana de la apercepción trascendental no estaría movida esencialmente por una determinación del yo cual substancia, sino en tanto experiencia de sí, la cual es refractaria a su homologación en una representación (2002, p. 340).

Queda claro, en virtud de lo dicho, que la mismidad ha de ser pensada, siguiendo a Heidegger, en relación con la estructura unitaria del cuidado. Empero, determinar al sí-mismo solo como un fenómeno en deuda con el cuidado nos parece insuficiente, a saber: a) la mismidad se ejerce como contramovimiento del uno-mismo, pero no es un contra-movimiento más, sino una manera excelsa, a partir de la resolución precursora, de asumir lo que «uno» es; b) el carácter de lo que somos es muy complicado sacarlo a la luz. Tal es así que, precisamente, aquí reside el meollo de la cuestión: si la mismidad la entendemos como el fundamento de toda asunción de la propiedad existencial ¿qué consecuencia se derivan de ello? ${ }^{15}$; c) la mismidad debe ser tematizada como aquel fundamento que resignifica y reinstituye un dominio de sentido sobre los momentos co-originarios y relativos al ser-en-el-mundo, no como un momento aislado, formado quidditativamente. Por eso, lo que es asumido, siempre ya, es el poder-ser arrojado que es el Dasein. Sin más.

Por ello, siguiendo el hilo conductor de la experiencia del sí-mismo, Heidegger habla de la «estabilidad del sí mismo» [Selbst-Ständigkeit], para identificarlo, de algún modo, con la resolución precursora. ${ }^{16}$ Sin embargo, esta analogía no le hace justicia a la función fundante de la mismidad, pues, aunque se acepte, con Heidegger, que la mismidad es el momento de asunción de un poder-ser propio y advenido a través de la resolución precursora, la cosa cambia cuando, en esta relación mismidad-resolución precursora, se versa sobre los rendimientos últimos de la ejecución ejercida por mor de esta misma resolución: un estado de apropiación, reinstauración y/o re-significación que es, en cada caso, algo que remite a un si-mismo/mismidad. Esto significa, entre tanto, que lo abierto por tal resolución precursora es algo más que la indicación existentiva de la posibilidad de un ser-total del Dasein, sino que, si la resolución remite inexcusablemente al sí-mismo, como su horizonte de comprensión, lo que se abre es siempre ya nuestra (mi) existencia como un

15 Al respecto Heidegger manifiesta (2002, p. 284): «¿Y cómo es el Dasein este fundamento arrojado? Únicamente proyectándose en posibilidades en las que está arrojado. 'El sí-mismo que, como tal, tiene que poner el fundamento de sí mismo, nunca puede adueñarse de éste $y$, sin embargo, tiene que asumir existiendo, el ser fundamento'» [Das Selbst, das als solches den Grund seiner selbst zu legen hat, kann dessen nie mächtig werden und hat doch existierend das Grandsein zu übernehmen]. Cursivas nuestras. Seguimos la traducción de J. Rivera (2006, p. 300).

16 (2002, p. 322): «La estabilidad del sí-mismo no significa existencialmente otra cosa que la resolución precursora. La estructura ontológica de la resolución precursora 'revela la existencialidad de la mismidad del sí-mismo'» [enthüllt die Existenzialität der Selbstheit des Selbst $]$. Cursivas nuestras. Seguimos la traducción de J. Rivera (2006, p. 338). 
proyecto arrojado que nos (me) concierne, lacera profundamente. No es de extrañar, en cambio, que investigaciones actuales realicen enfatizaciones sobre la experiencia del sí-mismo, en relación con la fenomenología en primera persona, o, desde otro lado, al amparo de unos análisis lógicos-lingüisticos, de corte analítico, sobre los usos de los pronombres personales, etc (Zahavi 2013 y 2005). Estas consideraciones, aunque pudieran ser importantes, son excéntricas a nuestra inquietud filosófica, la cual quiere centrarse, consciente de sus propias limitaciones, en el modo en el que la mismidad, además de ser tematizada por Heidegger como una manera de estabilidad -y, por tanto, desde ciertas resonancias hacia determinaciones adyacentes a la libertad, o a cierto ideal de autonomía-, radica en una función fundamental sobre la constitución del cuidado, en calidad de unidad extática de la temporalidad. Por tanto, en lo que sigue, se intentará mostrar cómo es posible que la mismidad dote de unidad al cuidado.

\section{EL SER-FUTURO Y EL HABER-SIDO DE LA MISMIDAD: LA TRASCENDENCIA DEL DASEIN}

El planteo de partida es que el cuidado es solo acreditado como fenómeno de sentido. Esto significa que el cuidado se muestra de manera no tan solo ejecutiva, sino como condición de posibilidad de la comprensión de todo ente, como el «dónde lo cual» [Woraufhin] algo puede ser mostrado en tanto algo y, por ende, articulado en su «significatividad» [Bedeutsamkeit]. De este modo podemos manifestar: a) el cuidado es dado como condición de posibilidad de ciertos comportamientos ligados al Dasein, como su fondo posibilitador; b) esto, que se hace temático a través del cuidado, es una modalidad emparentada en exclusividad por la «transparencia» [Durchsichtkeit], entendida como un modo ejecutivo de comprender que tiene un compromiso por dar cuenta del símismo más propio. A partir de estos dos horizontes, se tendrá que ver qué rol juega la mismidad, dentro del opus magnum heideggeriano, a fin de dar cuenta del cuidado, desde los dos momentos explicitados al inicio de este artículo, a saber: en relación con la resolución precursora, el momento de la anticipación de la muerte, su saberse finito, y el momento de hacerse cargo de tal finitud, mediante el ser-culpable.

\section{1. MISMIDAD Y EL PRE-CURSAR EL MORIR.}

Queda claro que el Dasein se pre-cursa en su posibilidad porque es un ser «futurizo» [zukünftig], es decir, tiene que hacerse en posibilidades, distenderse en un proyecto, pero siempre es él-mismo quien se pone en juego. En este sentido, pre-cursarse significa que el Dasein es posible en tanto «dejar-se-venir-haciasí-mismo» [Sich-auf-sich-zukommenlassen]. Sin este carácter autorreferencial del pre-cursar, en nuestra opinión, no sería posible tal anticipación, pues, entre 
tanto, las posibilidades futuras serían tan solo posibilidades en abstracto, a la manera en la que la semilla tiene la posibilidad de ser fruto. De este modo, como hemos dejado ver en otras investigaciones, es el ser-para-la-muerte el que dona y provee el fondo afectivo, relativo a la facticidad, el cual hace posible que las posibilidades se tornen una cuestión relativa al sí-mismo. Por eso, pre-cursar y ser-para-la-muerte están emparentados, pues ambos proveen el código de presentación de la posibilidad del Dasein.

\section{2. MISMIDAD Y HACERSE CARGO DEL SER-CULPABLE.}

Ser-culpable no significa llevar una carga, como si arrastrásemos remordimientos. Es, en cambio, empleado por Heidegger para rehabilitar el sentido latente de todo proyecto: el «estado de arrojado» [Geworfenheit], siendo que lo que se ha de «asumir» [übernehmen] es lo que siempre ya somos, o sea, la entraña temporal de la existencial: su finitud patentizada por el ser-parala-muerte. Ahora bien, la experiencia de la mismidad reinstaura un sentido sobre la facticidad, la cual pudiera ser entendida bajo criterios relativos a la «heteronomía», ya que, pudiera parecer que la facticidad es tan solo el ámbito de lo que ya esta ahí, antes de nosotros, como las tradiciones, o la historia, etc. Que la posibilidad, además de constituir un proyecto, sea arrojada, significa que todo ser «futurizante» es un ser que re-toma lo que ya es: «Solo en tanto que el Dasein es en general como yo soy sido, puede ir futurientemente al encuentro de sí mismo en un retorno» (2002, p. 326). Pero este retorno es solo posible porque está pivotando la mismidad como su condición de posibilidad en la manera de un «retomarse a sí mismo» [Wiederholung ihrer selbst].

\section{3. LA TRASCENDENCIA DEL DASEIN: BREVE ESBOZO.}

¿Cómo tematizar esta función que juega la mismidad en nuestro artículo filosófico? Tenemos, pues, que pensar la mismidad como un elemento religado al ámbito del sentido, es decir, tiene que ver con las condiciones de posibilidad de una re-significación del ámbito de la venida a la presencia, de la apertura a la comprensión, de aquello en lo que el Dasein, siempre ya, se relaciona en calidad de ser-en-el-mundo. La mismidad produce que este comportarse, en relación con el mundo, se vea potenciado en su aspecto autorreferencial, y que, por ejemplo, el mundo circundante no aparezca como una totalidad de entes que «están ahí» [vorhanden], sino como consecuencia de un poder-ser (existente/humano) que se ocupa de ellos. Justamente esto es lo que pensamos que llama Heidegger «situación» [Situation], una reinstitución autorreferencial a través de la cual el mundo, en su totalidad, aparece en homologación con el 
ser de este Dasein. Como se echa de ver, la posibilidad futura que retorna solo es posible bajo la empresa de llevar a cabo lo abierto en la situación:

De tal manera este fenómeno unitario, como futuro-que-está-siendo-sido, presenta lo que nosotros llamamos temporalidad. Sólo en la medida en que el Dasein está determinado por la temporalidad, hace posible para sí mismo el modo propio del poder-ser-entero de la resolución precursora. La temporalidad se revela como el sentido del propio cuidado (2002, p. 326).

Heidegger entiende, según lo abierto en el fragmento de arriba, que la situación es una modalidad en la que se abre un horizonte extático de tiempo, entendido como cuidado, el cual es extático no en virtud de su determinación como sucesión temporal entre pasado-presente-futuro, sino, más bien, a causa de la co-originariedad de sus momentos: pre-cursar (futuro), habersido (pasado) y ser-dentro-de (presente), todos ellos, vistos en unidad, han de ser entendidos, ahora de manera más clara, como cuidado (2002, p. 329). Pero ¿qué tiene, entonces, que ver esto con nuestros intereses investigadores en torno a la mismidad? ¿cómo puede incardinarse, sobre el fenómeno de la temporalidad del Dasein, el fenómeno de la mismidad? Tales preguntas son arduas, pero deben ser afrontadas y respondidas con seriedad. Para ello, es menester un tratamiento de la trascendencia del Dasein (Heidegger 1979, pp. 135-286), la cual radica en el fenómeno de la comprensión del ser, ya siempre sostenida por este ente. Ahora bien, la trascendencia, justo en este punto, debería ser tematizada como un desarrollo específico de la temporalidad, en el sentido de que lo temporizado, en la temporalidad que surca al Dasein, se muestra en la forma de lo ekstatikon por excelencia (Heidegger 2002, p. 329). No es de extrañar, por ende, que Heidegger tematice la temporalidad de carácter extático a partir del modelo de la horizontalidad, pues ya siempre lo abierto por la temporalidad es una dimensión relativa a un horizonte en la que el ser del Dasein, yendo más allá del ente por mor de su señalamiento radicado en la (pre-)comprensión del ser y en la «cuestionabilidad» [Fraglichkeit] de su existencia, lo trasciende, ejecutando, asumiendo su modo de ser, en y desde el cuidado que rezuma su modo peculiar de «temporalizarse»: este horizonte extático-temporal, propio del cuidado, conglomera el futuro, en la forma del «hacia sí» [auf sich zu]; el pasado, en la manera de estar «de vuelta a» [zurück zu]; y el presente, en la situación abierta del «hacer comparecer algo» [Begegnenlassen von] (2002, p. 329). Para resumirlo: la trascendencia del Dasein, como el momento cinético recogido en el salirde-sí, hace coparticipe, desde un horizonte, los tres éxtasis temporales, los cuales quedan materializados y concretizados mediante el rol constituyente que conforma el cuidado. Al manifestar esto, Heidegger, en nuestra opinión, no puede mostrar la unidad si no es acudiendo al horizonte conformante de la 
mismidad, pues el cuidado ha de ser, siempre ya, cuidado de sí -a pesar de la redundancia-, como, del mismo modo, cuidado del mundo-entorno y cuidado del mundo-compartido. De lo contrario, el Dasein se abriría extáticamente, en su cuidado, de forma parcial, fragmentaria, sin irle su ser en ser (existir). Nota bene, la tematización de la temporalidad del Dasein, casi recíprocamente, da buena cuenta de por qué no la mismidad ha de ser asumida bajo un carácter cósico. La mismidad del Dasein es, por ende, una apropiación cinética que tiene como fin sacar a relucir una posibilidad en armonía con la idiosincrasia existencial de este ente. Que el Dasein sea una temporalidad signada como cuidado significa que existe en la manera de siendo-sido, nunca como un ente intramundano.

\section{CONCLUSIONES: EL CARÁCTER ORIGINARIO DE LA MISMIDAD.}

Con todo, el desarrollo de nuestro análisis fenomenológico no ha mostrado -aunque sí señalado- de qué modo la mismidad es un fenómeno originario (Corti 2006, pp.152-178), y su relación con la resolución precursora y la temporalidad. La exigencia sobre el origen debe ser buscada en cuanto se intenta retrotraer los fenómenos a su ámbito de mostración integral. Que la mismidad sea originaria significa, por un lado, que a partir de ella el modo de ser del Dasein es articulado en su integridad y, por otro, que tal mismidad es constante/estable/retenida en su relación con todo tipo acto que trate de llevar a término la temporalidad, ora impropiamente, ora propiamente ¿Subyace, dejándose ver, la mismidad en toda forma en la que «se realiza» [vollzieht sich] la existencia? El planteo de Heidegger, a través de una extracción de la forma del movimiento transcrito por el modo como se ejecuta la trascendencia, consiste en que tal fenómeno subyacente, de alguna manera, es la temporalidad, la cual se hace originaria porque es condición de posibilidad del ser del Dasein mismo. Bien es cierto que el fundamento abierto por la temporalidad no es más que una forma de temporalización -no de quiddidad-, o sea, de tomar cuerpo y de hacer concreto un posible modo de asunción de una posibilidad que es el Dasein mismo. Pero esta forma de asumir, que es el modo en el que se temporaliza la temporalidad que somos, siempre tiene como presupuesto la mismidad, ya que, no lo olvidemos, fue la resolución precursora la que nos puso ante la apertura de la «situación» [Situation], a fin de hacer valer la integridad del Dasein como temporalidad. Si esto es cierto, en el precursar no está solo presente el ser-para-la-muerte, sino también la mismidad, que hace posible toda anticipación de las posibilidades de nuestro poder-ser (existencia). En otras palabras, el Dasein se anticipa no solo por las implicancias que el haber-sido tiene sobre todo proyecto basado en el poderser, sino porque el haber-sido nos concierne, remite lacerantemente a algo que somos, comprendiéndolo afectivamente como nuestro. 
El hecho que muestra, al parecer, que Heidegger no tiene todo esto muy claro, se debe, empero, a la constante analogía producida entre las expresiones «temporalidad originaria» [ursprüngliche Zeitlichkeit] y «temporalidad propia» [eigentliche Zeitlichkeit] (Corti 2006, p. 158). Ambas expresiones no pueden ser lo mismo, sino que una presupone a la otra.

En definitiva, sin riesgo a equivocarnos, afirmamos que es la temporalidad propia la que supone un modo de concretización fenomenológica sobre la temporalidad originaria. La adjetivación de fenomenológica sobre la concretización no es baladí, sino indica que la temporalidad propia es todo aquel intento de llevar a cabo una ejecución que no modifica, no sustrae el elemento de origen, el fenómeno desde su horizonte de sentido, sino que lo religa ineludiblemente. ${ }^{17}$ Pero, en virtud de lo dicho, la temporalidad originaria es la que hace referencia a la mismidad como momento eminente por el cual el Dasein se abre a sí mismo, siendo que la temporalidad propia es un modo de preservar asumiendo lo abierto a partir de la mismidad, y todo ello sin distorsionar el carácter de apropiación de tal sí-mismo, hendido y descubierto por la temporalidad.

\section{REFERENCIAS BIBLIOGRÁFICAS.}

BLATTNER, W. (1999). Heidegger's temporal Idealism. Cambridge: Cambridge University Press.

CORTI, A. (2006). Zeitproblematik bei M. Heidegger und Agustinus. Würzburg: Königshausen \& Neumann.

GARRIDO-PERIÑÁN, J. J. (2019a). «La aportación no-apofántica de la disposición afectiva y la mismidad del Dasein: análisis fenomenológico a partir del momento estructural ser- en», Pensamiento: Revista de Investigación e Información Filosófica 75 (285), pp. 887-911.

GARRIDO-PERIÑÁN, J. J. (2019b). «Vinculabilidad entre Cuidado y Mismidad en los $\S \S 39-42$ de Ser y Tiempo: Heidegger y la mismidad del Dasein», Alpha: Revista de artes, letras y filosofía 49, pp. 159-175.

GARRIDO-PERIÑÁN, J. J. (2019c). «El rol protagónico de la mismidad del Dasein en el momento de transición entre la I y II Sección de Ser y Tiempo: atenencias metódicas y temáticas», Bajo palabra: Revista de Filosofía 22, pp. 411-206.

17 Entonces, por otro lado, la temporalización de la temporalidad originaria en la impropia sería una derivación, la cual modifica, transformando, a la manera de la reificación, lo asumido (Heidegger 2002, pp. 346-349). Para un desarrollo de la cuestión: (Blattner 1999, pp. 89-126; Gelven, 1989; Greisch, 1994; Pasqua, 1993 y Másmela, 2000). 
GARRIDO-PERIÑÁN, J. J. (2019d). «La pregunta por el quién del ser-con: Heidegger en su Ser y Tiempo», Anales del Seminario de Metafísica 36 (1), pp. 175-200.

GARRIDO-PERIÑÁN, J. J. (2019e). «La fuerza configuradora de la imaginación en el tiempo de la auto-afección de la mismidad: Kant y Heidegger», Veritas: Revista de Filosofía y Teología 43, pp. 9-28.

GELVEN, M. (1989). A commentary on Heidegger's Being and Time: New York: Northern Illinois University Press.

GREISCH, J. (1994). Ontologie et temporalitè: Esquisse d'une interprétation intégrale de Sein und Zeit. Paris: PUF.

HEIDEGGER, M. (2006). Ser y Tiempo. Trad. Jorge Eduardo Rivera. Madrid: Trotta.

HEIDEGGER, M. (2002). Sein und Zeit. Tübingen: Max Niemeter.

HEIDEGGER, M. (1979). Metaphysische Anfangsgründe der Logik im Ausgang von Leibniz. II. Abteilung, Band 26. Frankfurt a. M.: Vittorio Klostermann.

OPILIK, K. (1993). Transzendez und Vereinzelung. Zur Fragwürdigkeit des Transzendentalen Ansatzes im Umfreis von Heideggers 'Sein und Zeit'. Freiburg: Karl Alber.

PASQUA, H. (1993). Introduction aà la lectura de Etre et temps de M. Heidegger. Lausanne: L'Age d'Homme.

MÁSMELA, C. (2000). Martin Heidegger: el tiempo del ser. Madrid: Trotta.

ZAHAVI, D. (2013). Self, no self?: perspectives from analytical, phenomenological, and Indian traditions. Oxford: Oxford University Press.

ZAHAVI, D. (2005). Subjectivity and selfhood. Cambridge: MIT Press.

JuAn José Garrido Periñán es profesor sustituto interino en el Departamento de Estética e Historia de la Filosofía de la Universidad de Sevilla. Miembro fundador y Secretario del Archivo-Heidegger de la Universidad de Sevilla (Delegación Martin Heidegger-Archiv de Meßkirch) y miembro investigador del Proyecto I+D "DiCiOr" (FFI2017-83770-P) que es financiado por el Ministerio de Ciencias y Universidades del Reino de España.

Lineas de investigación:

Fenomenología y hermenéutica de la existencia, fenomenología del sí mismo en el pensamiento de Heidegger, metafísica y ontología

Publicaciones recientes:

(2019). «La aportación no-apofántica de la disposición afectiva y la mismidad del Dasein: análisis fenomenológico a partir del momento estructural ser- en», Pensamiento: Revista de Investigación e Información Filosófica 75 (285), pp. 887-911. 
(2019). «La fuerza configuradora de la imaginación en el tiempo de la auto-afección de la mismidad: Kant y Heidegger», Veritas: Revista de Filosofía y Teología 43, pp. 9-28.

Correo electrónico: jjgarper@us.es 
